\title{
Instrumentation Selection Analysis for an Air Compressor System and Enhancement Proposal by Sensors Based on Nanostructures
}

\author{
Jesús Alan Calderón Chavarri, ${ }^{1, *}$, Luis Ricardo Chirinos García ${ }^{2}$, Enrique José Barrantes Peña², \\ Ronald Eduardo Mas Bautista ${ }^{2}$
}

${ }^{1}$ Department of Applied Nanophysics, Ilmenau University of Technology, Ilmenau, Germany

${ }^{2}$ Energy Laboratory, Pontifical Catholic University of Peru, Lima, Peru

\section{Email address:}

natmarnot@gmail.com (J. A. C. Chavarri), lchirin@pucp.edu.pe (L. R. C. García), ebarran@pucp.edu.pe (E. J. B. Peña), rmas@pucp.edu.pe (R. E. M. Bautista)

${ }^{*}$ Corresponding author

\section{To cite this article:}

Jesús Alan Calderón Chavarri, Luis Ricardo Chirinos García, Enrique José Barrantes Peña, Ronald Eduardo Mas Bautista. Instrumentation Selection Analysis for an Air Compressor System and Enhancement Proposal by Sensors Based on Nanostructures. Journal of Electrical and Electronic Engineering. Vol. 6, No. 3, 2018, pp. 88-93. doi: 10.11648/j.jeee.20180603.14

Received: June 6, 2018; Accepted: July 16, 2018; Published: August 29, 2018

\begin{abstract}
Air compressor systems are widely used in industry due to increase pressure level of gases in order to solve many tasks such as in air supply, or just to transmit mechanical movement. For this reason, instrumentation selection is quite important because correct and precise physical variables are necessary requirements to get a uniform pressure level transmission. The physical variables of an air compressor system were studied by its instrumentation selection based on sensors/actuators physical parameters such as response time and robustness. Furthermore, it was shown the compressor system performance enhancement while instrumentation is based in nanosystems like nanosensors and nanoactuators.
\end{abstract}

Keywords: Sensors, Thermodynamic Systems, Nanosensors, Compressor System

\section{Introduction}

Thermodynamic variables in air compressor systems were evaluated many years ago through electronic devices without enough memory capacity such as "Logic Gates", first evolution of microprocessors like "Atmel and Arduino" families obtained results to make different calculations to get a kind of autonomy by them. Nowadays, computer systems achieved successful advances, as it is given for example by "National Instruments" with controllers "MyRio and CompactRio" $[1,2]$. Therefore, with that sophisticated hardware and software strategies is possible to integrate traditional sensors and actuators on systems, in order to get the balance among acquisition data, response actions over fast response time systems.

Nevertheless, there are equipment such as thermodynamic systems which have multiple variables to be monitored and to be processed by a controller with objective to give a fast response and to get a control. One of this kind of systems were studied in this research: "Air compressor system PLINT" from Energy Laboratory of PUCP. When this equipment is in presence of disturbances, furthermore when it has problems with sensors and actuators due to vibrations during operations, there is necessary good programming strategies in contemplation of achievement correct information to get a right integration on the compressor system to study all thermodynamic variables.

On other side, there is proposed in this research to replace traditional sensors and actuators by sensors/actuators based on high ordered nanostructures, it because to obtain fast measurement response time, fast response actions over the compressor system. It means, through new materials characteristics it is possible to use new kind of sensors and actuators that can work at fast responses and get the mechatronic integration without complicated programming strategies, which help to save memory space to be used in another tasks of the main system. 


\section{Theoretical Descriptions}

It is described a schema of the system in figure 1, where it is represented operation of the PLINT air compressor system, that needs an excitation signal to start to work, which is given by $U$. It is suggested to represent all the system as dependence of $U$ like input variable, due to the fact that through this input excitation signal is possible to give energy to the whole compressor system, by other side "disturbances" must to be always considered in the analysis, owing to that can appear through vibrations, electromagnetic noise, impedance variations among sensors and actuators of the system. Also, for this research, it was proposed "Pressure" and "Flow" as output variables of the system due to short response time compared with temperature response time (for all the flow road to through the compressor) therefore it is possible to show response of the system while to be waiting all the steady state in parallel to get mathematical models by Pressure and Flow previously.

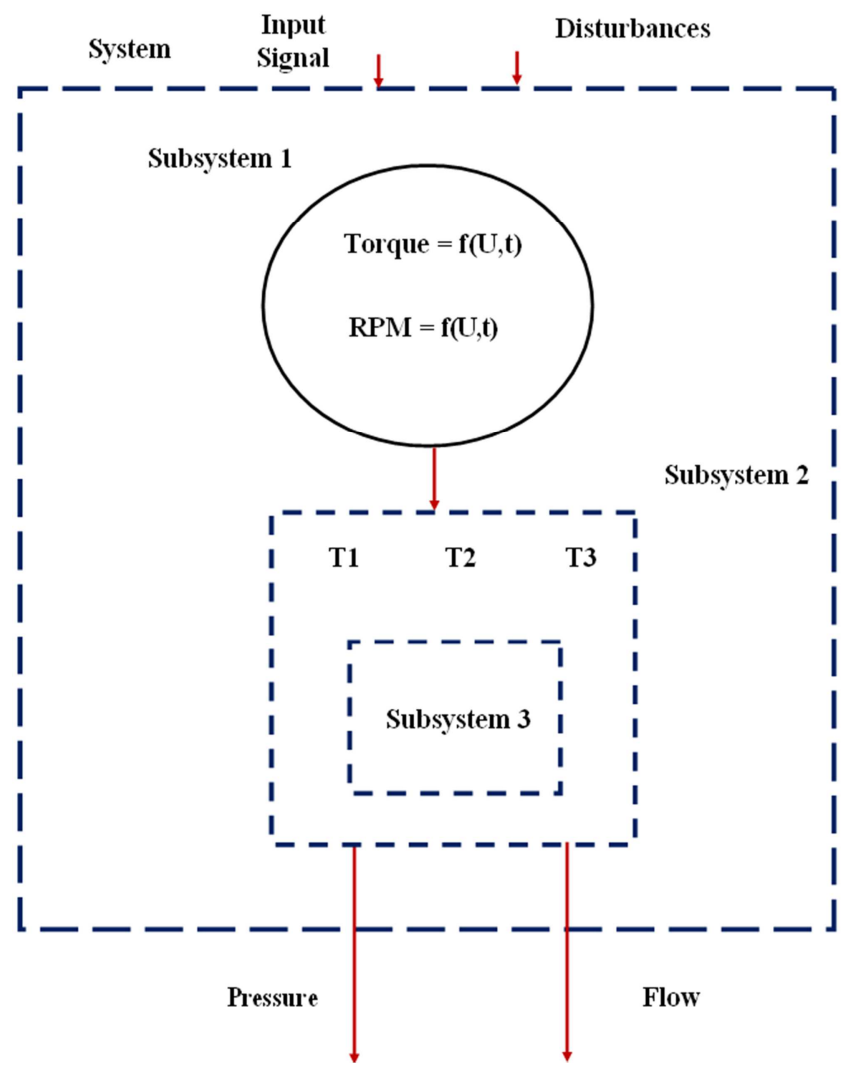

Figure 1. Air compressor system schema.

For this research, it was proposed to divide the main system in 2 subsystems: "Subsystem 1" that is composed by "Torque" and "RPM" of the DC motor which transforms electrical energy to mechanical movement to share with the main system. For this reason, this subsystem is considered as "Mechanical subsystem" of the Air Compressor. On the other hand, the "Second subsystem" is proposed by thermodynamical variables joined to integrate all thermodynamic information, in which temperature in compressor entrance, inside the compressor, and compressor outlet are considered. Furthermore, it can be considered a subsystem inside the second, it is regarding to find thermodynamic variable connections between air pressure inside air camera and flow changes through valve state in order to get balance to correlate thermodynamical variables and study the system.

\section{Compressor System Application}

The PLINT compressor system (our equipment) is composed by a DC motor that receives electrical energy to transmit movement and torque to the air compressor, that let to get pressure changes on the air flow, furthermore temperature changes on the road of compressor, also it is possible to control the pressure through a regulation of valve connected to the air camera. Figure 2 shows the thermodynamic system to study.

It must be known that with a selected instrumentation, thermodynamic variables from the PLINT compressor system can be studied. For this reason, accurate sensors, actuators and depending system response also disturbances, a controller must be selected, it is also important a controller selection to solve task such as monitoring information from the thermodynamic system, some control tasks, each of them connected by an operating system to execute the main algorithm. Notwithstanding, when disturbances given as vibrations or impedance changes among sensors, actuators connections with the controller and system, so good and fast strategies in the algorithm are necessary to give a reaction in order to keep the balance during acquisition information from the system.

However, whether it can be found and replaced traditional sensors and actuators by equivalent of them which could have faster response time and good impedance while measuring or giving response information on the system, so acquisition information could not have much dependence on the main algorithm, it means that thermodynamic data would not suffer so many processing that in real it can modify part of the real information of the system. Hence, new materials to create new sensors and actuators give wide advantages to get a robust mechatronic integration, as it is proposed in this work.

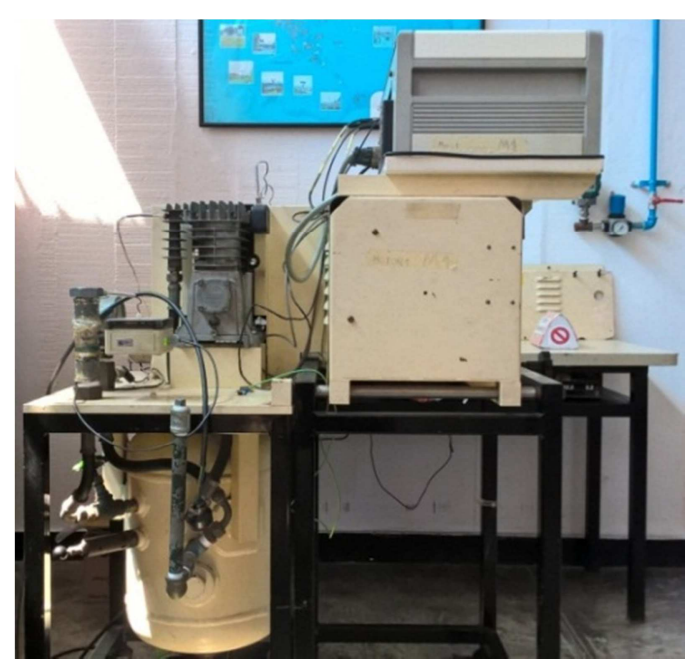

Figure 2. PLINT, the air compressor system. 


\section{Instrumentation}

The air compressor system is a design from PLINT Company, that needed to be remodeled or modernized due to its older interface to interact between "input variables: electrical excitation input signal (main input signal in voltage), RPM (Revolution Per Minute); output variables: pressure (inside camera to store the air in bar), temperature (three values which are at the entrance of the compressor, inside camera and at the exit of this in Celsius degrees) and finally the air flow (in liters per minute)". All these variables were registered and processed by a controller from "MyRio of National Instruments". Nevertheless, in some cases it was designed "Virtual Sensors" when some of the thermodynamical variables were not possible to be register, it because the PLINT compressor system (our plant) is very older and its sensors too; therefore, under that situations we designed a predicted algorithm to work as virtual sensors and to not loss information [3].

\subsection{Temperature Sensor}

The PLINT air compressor system has thermocouples type $\mathrm{K}$ as temperature sensors. It was used an Electrical Current transductor in order to compensate, amplify and linearize temperature signal, furthermore It was filtered temperature signals considering AWGN (Additive Withe Gaussian Noise) as main noise coupled during the measurements [4].

\subsection{Pressure Sensor}

It was worked an electrical pressure sensor and compared with a manometer (both are components of the PLINT air compressor system), the trouble in this remodeled system was that pressure sensor was damaged for the range of work
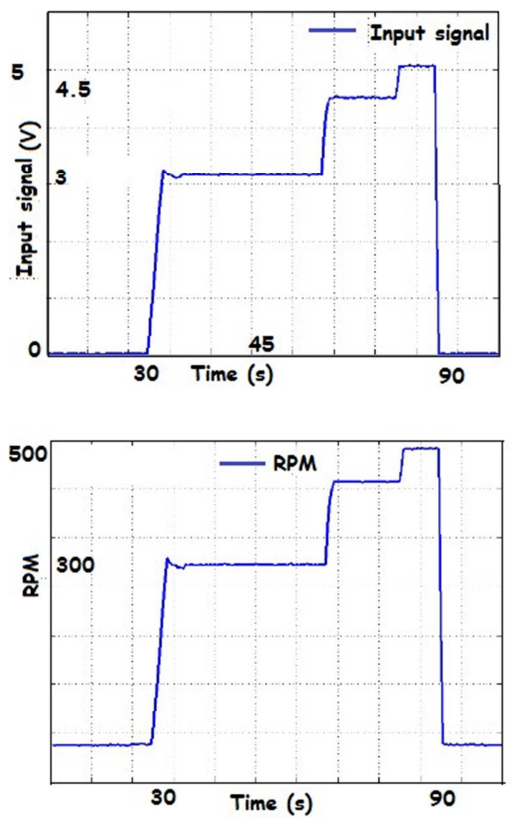

from 2Bar to 4Bar. Therefore, it was analyzed linear correlation with another thermodynamic variable (air flow), with which was estimated the pressure measurement through an observer such as a virtual sensor.

\subsection{Air Flow Sensor}

As a flow sensor, it was used a rotameter with a display indicator own from the PLINT compressor system, that was not correctly fixed to its exit channel (maybe because many years to be used this system) for this reason it generates disturbance during data acquisition. Therefore, the software support is a good solution (algorithms to design different filters, observers or virtual sensors).

\section{Data Acquisition}

The compressor system was tested by monitoring and storing all its measured variables in order to analyze the behaviour of its sensors, even though as it was described, in many cases it was necessary to design virtual sensors to get the thermodynamical variables. In figure 3 , it is shown the input excitation signal (that is correlated with the RPM, because of this excitation signal is the electrical voltage response in order to get excitation as RPM in the motor which gives energy to the air compressor), furthermore, it is shown the RPM signal from the motor axis, torque signal produced by the motor and the pressure signal produced by the air stored inside camera. As it is shown in figure 3, torque signal needs more than a filter in order to be reconstructed (that was achieved through a virtual sensor also when its sensor could not get signal). By other side, the pressure sensor also was filtered and analyzed by the virtual sensors carefully when the system was between 2 bars to 4 bars.
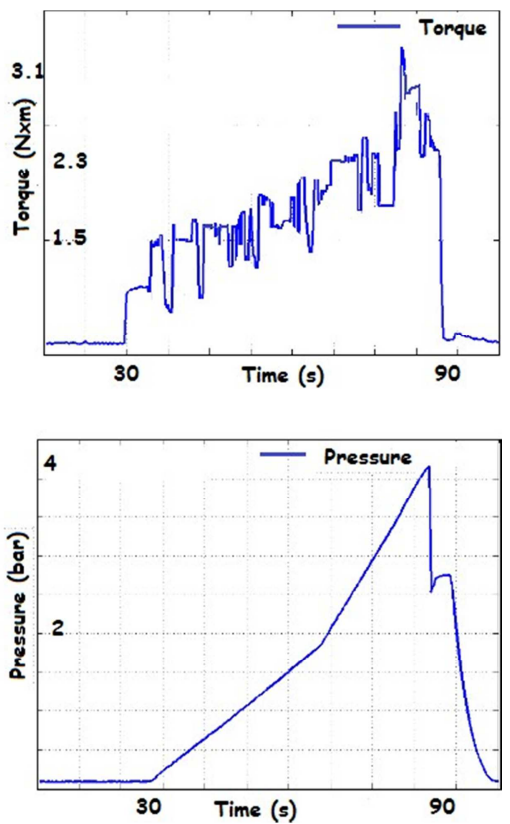

Figure 3. Measured variables: Input signal, Torque, RPM and Pressure.

The measured temperatures are shown in figure 4 (which needs a predictive filter in order to improve measured data under vibrations) at the entrance of the air compressor system (Temperature 1), the temperature inside the camera (Temperature 2) 
and the temperature in the exit channel of the air compressor (Temperature 3) furthermore, it is depicted the air flow signal, which also was a result of our virtual sensors (a consequence of a predicted observer) [3].
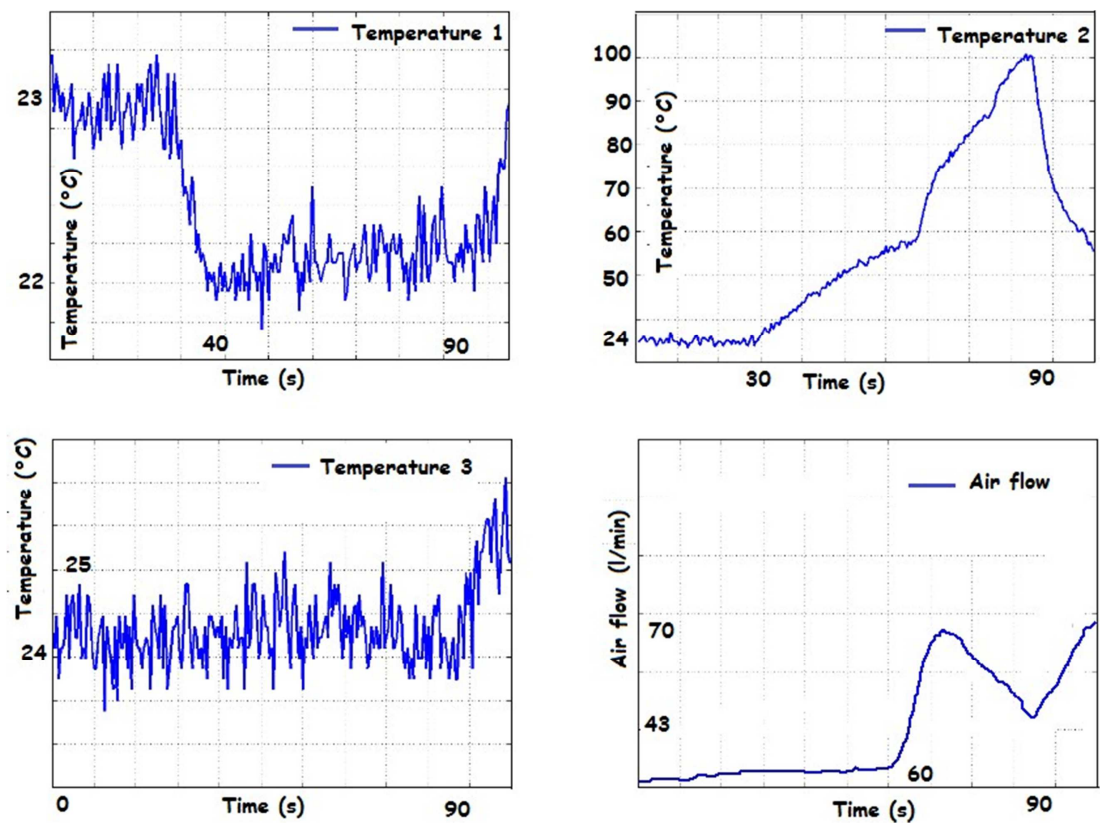

Figure 4. Measured variables: Temperatures and Air flow.

\section{Proposal}

Sensors based in high ordered nanostructures arrays can be a good support to measure physical variables such as the thermodynamic variables which we are being studied in this work: "Temperature, Pressure and Air Flow", it is expected robustness and short response time because of the high ordered geometrical properties from nanostructures arrays. It was simulated transductors properties changes based in AAO (Aluminium Oxide) nanomembranes, which also can be the main support to elaborate nanostructures as nanotubes or nanowires in order to transform the measured physical variable to equivalent electrical variable (Voltage or
Electrical Current) by analysis of statical and dynamical response between that physical variable changes [4-7].

For this reason, in figure 5 is depicted a simulated response for a temperature sensor emulating this sensor elaborated by AAO template based on $\mathrm{TiO} 2$ (Titanium Dioxide) nanosystems (green color curve) which is compared with a traditional temperature sensor, a type $\mathrm{k}$ thermocouple $[4,8]$ (blue color curve). It can be verified that through simulations, temperature sensor based on AAO nanosytems is faster compared with a traditional temperature sensor, we expect that result by analyzing robustness in $\mathrm{TiO} 2$ high ordered nanostructures.

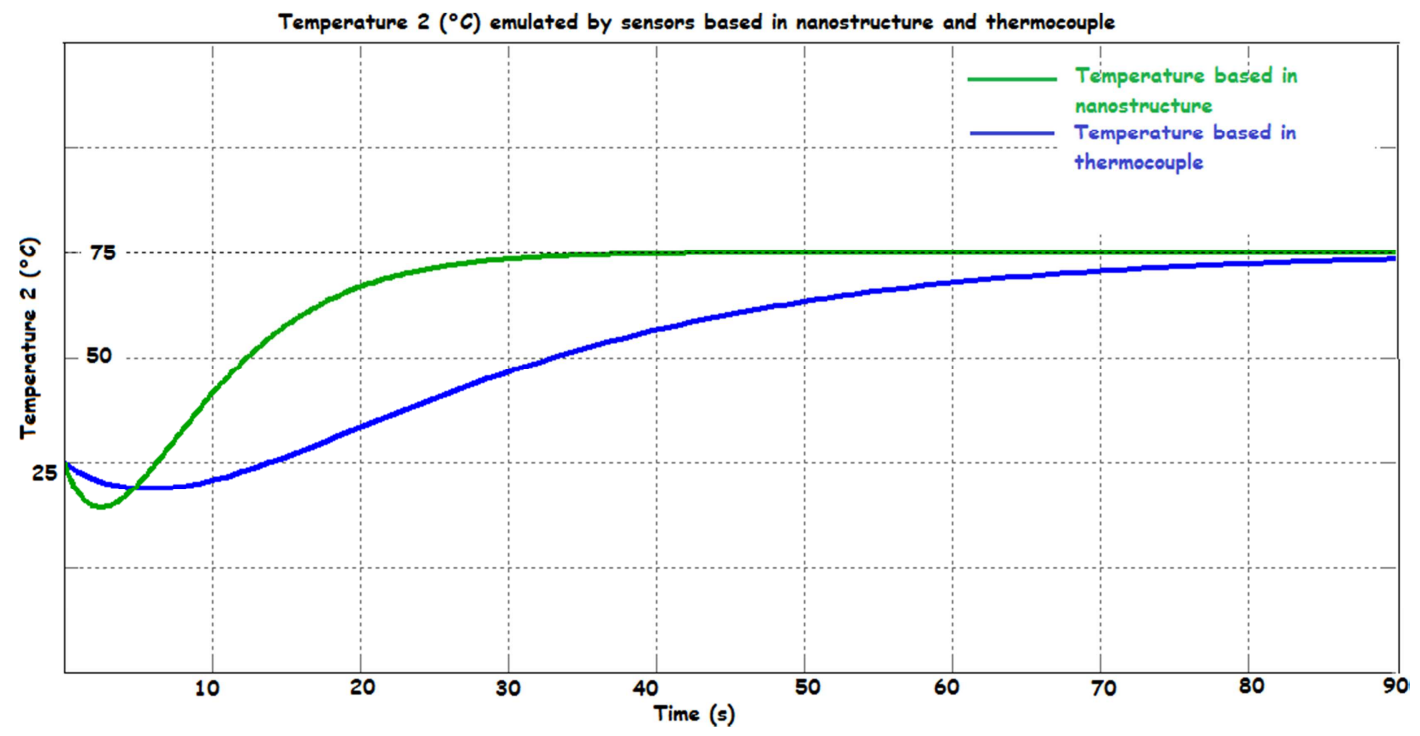

Figure 5. Temperature 2 emulated by sensors based in nanostructure and thermocouple. 
The necessity to get short response time compared with response time of process are so important to be achieved in order not to lose information of the process, such as for example figure 6 shows temperature changes for data registered from the PLINT compressor system, in which it is frequently to make mistakes by considering as first order temperature response from the system, so its delay is not analyzed; that is right system response time is so bigger than its delay (more than 100 its times) that can be corroborated analyzing the mathematical model.

Therefore, as it is shown in figure 6 , blue color curve represents temperature response system without delay considerations, at same time, red color curve gives information of the temperature system while it is included its own delay, by this reason it is simple to understand why is very important to look for faster response sensors, in that figure also it is depicted 2 temperature sensors response, by yellow color curve is represented temperature sensor response of the PLINT air compressor (controlled), that is faster enough than the pressure changes on the system [9]. However, problems come while fast changes disturbances appear on the system, such as vibrations caused due to operation of the PLINT compressor, in this context its own temperature sensor has not a fast response to get real information, that is one necessity which can be enhanced through proposal of this research "Sensors based on nanosystems" like it is described in figure 6 the controlled temperature by sensor simulated response based on AAO template (green color curve) and $\mathrm{TiO} 2$ high ordered nanostructure $[5,10,11]$.

As it can be verified by comparison every temperature curve, the best temperature sensor can be obtained by nanosystem due to faster response to get performance while monitoring information of the main system, also it can help in cases where it is not considered system delay owing to this sensor has enough time to response under perturbations even though system own delay. Furthermore, as it was described paragraphs above, robustness and fast response time obtained from sensors proportionate advantages to reduce computing time on the main system, it because its speed compared with traditional sensors (by this work it is achieved more than 4 times of them) so, it implicates to reduce computing programming cost on the main algorithm, in order to use it for another strategies such as to create observers on the main system. In this work, it was designed a monitoring algorithm, in which it was necessary to create an observer of the temperature data in order to measure (by reflection in determined range of work) air flow variable, also the Operating System was "Windows non Real Time", which means that the system did not has enough time to response under fast disturbances or changes (such as undesired temperature changes due to movement over valve not correctly fixed) as it was problematical but can be solved when are replaced by sensors based on nanosystems.

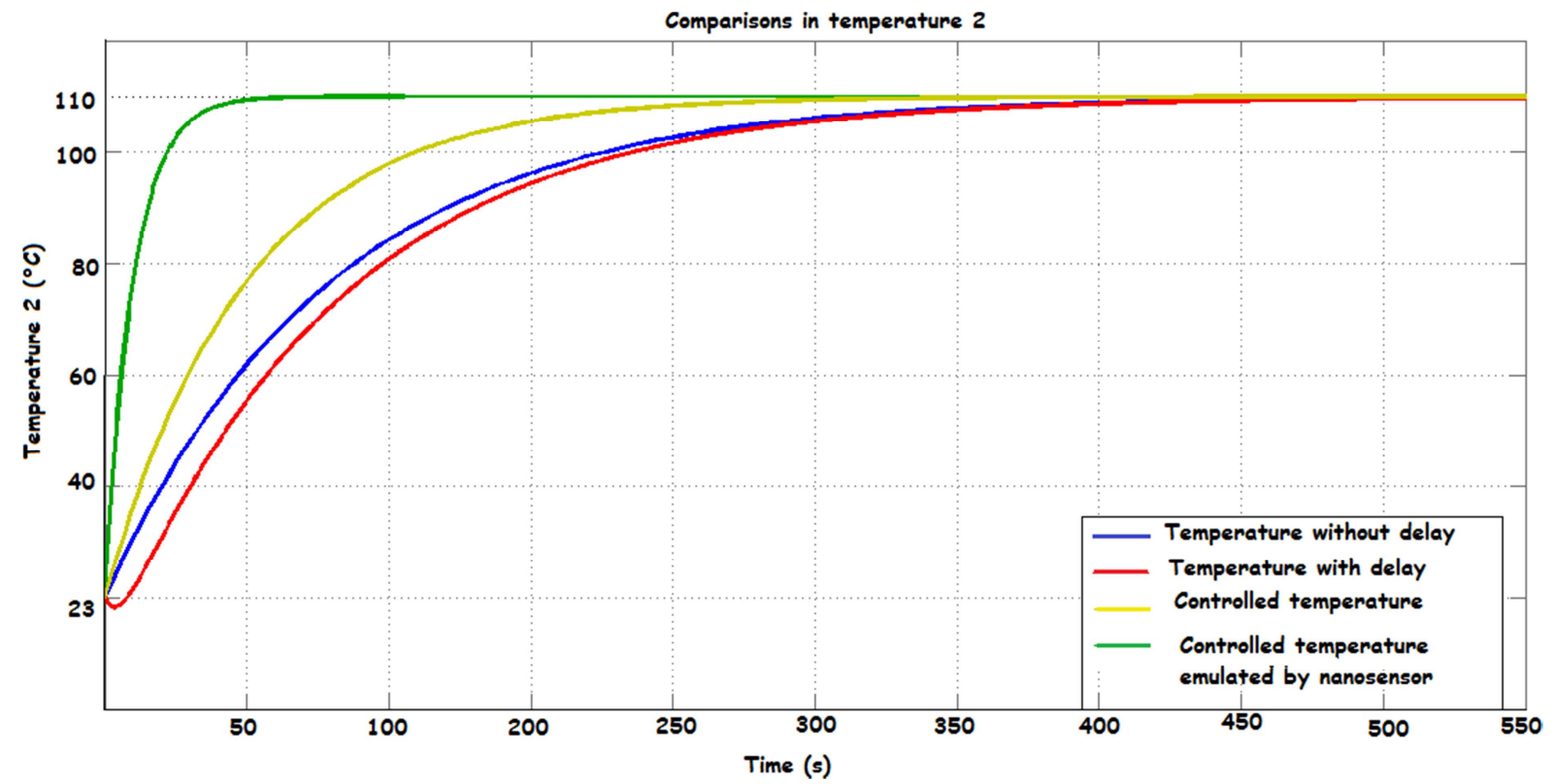

Figure 6. Comparisons between system, traditional sensors and sensors based on nanosystems.

\section{Conclusion}

In this work, it was studied the data acquisition of thermodynamic variables (pressure, temperature and air flow) by a PLINT air compressor system, also we propose some strategies on how to obtain and study data acquisition in order to give enough capability to get a mathematical model for the thermodynamic system, which cannot be identified successfully when there is not a correct measure.

Furthermore, it is suggested to capture information by the sensors when actuators and them are not enough faster than the thermodynamic system response. However, that strategy have computing cost which are not an advantage due to it means to generate troubles in response time under disturbances. For this reason, it is also proposed sensors and 
actuators based on high ordered nanostructures arrays because of their fast response besides their robustness under perturbations.

\section{Acknowledgements}

Furthermore, there is expressed kind greetings to Mr. John Lozano, Mr. Juan Lengua, Mr. Broni Huamaní and Mr. Eduardo Pichilingue because of all their support during experimental tests.

\section{References}

[1] N. Instruments, «User guide and specifications NI myRio1900». [En línea]. Available:

http://www.ni.com/pdf/manuals/376047a.pdf.

[2] N. Instruments, «NI CompactRIO High-Performance RealTime Controllers», 0611 2014. [En línea]. Available: www.ni.com/datasheet/pdf/en/ds-201.

[3] L. Wang, Model Predictive Control System design and implementation using MATLAB., London: Springer-Verlag, 2009.
[4] Honeywell, «Megopal Thermocouples, specifications», 1998.

[5] S.-h. Jo, «Fabrication and crystalization of AAO template for sensor applications», 2012.

[6] H. Yan, «Preparation and optical characterizacion of nanoporus templates as a basis for nanocontact arrays», 2012.

[7] Y. Lei, «Highly ordered nanostructures with tunable size, shape and properties: A new way to surface nano-pattering using ultra-thin alumina masks», 2007.

[8] Farnell, «Analog Devices: Monolithic Thermocouple Amplifiers with Cold Junction Compensation AD595», 1997. [En línea]. Available: http://www.farnell.com/datasheets/14024.pdf.

[9] L. Ljung, Modeling of dynamic systems, Prentice Hall, 1994.

[10] A. E. Pearson, «Aerodynamic Parameter Estimation Via Fourier Modulating Function Techniques», NASA, 1995.

[11] J. Klamka, «Controllability of dynamical systems», SpringerVerlag, 2008. 